\title{
Shape deformations in rough-surface scattering: cancellations, conditioning, and convergence
}

\author{
David P. Nicholls \\ Department of Mathematics, University of Notre Dame, Notre Dame, Indiana 46556 \\ Fernando Reitich \\ School of Mathematics, University of Minnesota, Minneapolis, Minnesota 55455
}

Received February 21, 2003; revised manuscript received August 27, 2003; accepted November 14, 2003

\begin{abstract}
We analyze the conditioning properties of classical shape-perturbation methods for the prediction of scattering returns from rough surfaces. A central observation relates to the identification of significant cancellations that are present in the recurrence relations satisfied by successive terms in a perturbation series. We show that these cancellations are precisely responsible for the observed performance of shape-deformation methods, which typically deteriorates with decreasing regularity of the scattering surfaces. We further demonstrate that the cancellations preclude a straightforward recursive estimation of the size of the terms in the perturbation series, which, in turn, has historically prevented the derivation of a direct proof of its convergence. On the other hand, we also show that such a direct proof can be attained if a simple change of independent variables is effected in advance of the derivation of the perturbation series. Finally, we show that the relevance of these observations goes beyond the theoretical, as we explain how they provide definite guiding principles for the design of new, stabilized implementations of methods based on shape deformations. (c) 2004 Optical Society of America

OCIS codes: $050.0050,050.2770,290.0290$.
\end{abstract}

\section{INTRODUCTION}

Analytical and numerical methods based on shape deformations have been widely used in scattering calculations since the original work of Rayleigh ${ }^{1}$ and Rice ${ }^{2}$ and have successfully led to new insights in a variety of applications, including acoustics, spectroscopy, optics, remote sensing, etc. Besides being simple to implement, these perturbative approaches generally lead, quite efficiently, to quality predictions within their domain of applicability. Indeed, it was these characteristics that prompted a number of investigations in the last 30 years, mainly in the area of scattering by rough surfaces, and that resulted in a variety of low-order theories (see, e.g., Refs. 2-9). The efficiency of these approaches naturally led to consideration of their applicability and accuracy; despite significant advances in the elucidation of these properties over the last decade, some important aspects remain unresolved.

The issue of applicability of shape-perturbation methods is, of course, closely related to the order of the perturbation expansion and therefore to its convergence properties. These properties, in turn, have apparently generated a controversy in the literature, dating back to the work of Meecham ${ }^{10}$ and Uretsky ${ }^{11}$ and further enhanced by the limited success of straightforward attempts at high-order expansions in numerical simulations (see, e.g., Refs. 12-22). A definitive answer to the question of convergence of shape-perturbation expansions was finally provided in Ref. 23, where it was proved that, for analytic scattering surfaces, the scattered fields are analytic functions of a deformation parameter. For the case of infinite (periodic) rough surfaces, this result asserts that the scattered field analytically continues to that of a flat surface, thereby guaranteeing the convergence of the series, at least for small perturbations. More importantly perhaps, in this case the theoretical results in Ref. 23 (subsequently illustrated in numerical simulations in Refs. 24-29) establish that the fields corresponding to a flat surface analytically continue to perturbations of arbitrary size. Of course, such perturbations may not be attainable by a simple Taylor-series sum; rather, alternative summation (i.e., analytic continuation) mechanisms may be necessary (see, e.g., Refs. 25 and 26).

The issue of accuracy and, more precisely, conditioning of shape-perturbation approaches, on the other hand, has remained largely unexplored. This is, in fact, apparently the case for all perturbative schemes, including alternatives such as the operator expansion (OE) method of Milder et al. ${ }^{30-38}$ In this paper, we address this issue as we uncover the precise mechanism giving rise to potential instabilities in classical shape-perturbation methods. As we demonstrate, these instabilities arise as a result of significant cancellations that occur in the recurrences underlying a typical shape-deformation scheme. Indeed, we show that these cancellations are precisely responsible for the observed performance of these methods, which typically deteriorates with decreasing regularity of the scattering surfaces. We further show that the cancellations preclude a straightforward recursive estimation of the size of the terms in the perturbation series, which, in turn, has historically prevented the derivation of a direct proof of its convergence. On the other hand, we also 
show that such a direct proof can be attained if a simple change of independent variables is effected in advance of the derivation of the perturbation series. In addition to providing a more straightforward path to convergence than that presented in Ref. 23, which we therefore hope will be more readily accepted by practitioners, these observations have immediate practical relevance, as they provide definite guiding principles for the design of new, stabilized implementations of boundary perturbation methods.

The rest of the paper is organized as follows. In Section 2 , we introduce our notation, and we briefly review some of the most commonly used shape-perturbation methods. In Section 3, we discuss the conditioning issues that we alluded to above, as we describe the way in which cancellations arise at both low and high orders of the recurrences. Section 4 is devoted to the convergence properties of the perturbation series. There we first explain how the cancellations preclude an iterative estimation of the terms in the series in the original variables. We then also show that a simple change of variables can be effected to implicitly account for cancellations, thus permitting a direct, inductive bound of the perturbation coefficients that delivers a convergence result. Finally, some concluding remarks are presented in Section 5, which include a set of guidelines for the design of improved numerical simulation strategies based on shape deformations.

\section{SHAPE-DEFORMATION METHODS}

As we mentioned, we shall be centrally concerned with stability properties of shape-perturbation methods in rough-surface-scattering calculations. For simplicity, we shall restrict our discussion to the scalar case in two space dimensions, although our observations and algorithms clearly extend to the full vector equations in threedimensional space; see the remarks at the end of this section. Here we first set up our notation and then briefly review some classical perturbative algorithms.

\section{A. Scattering by Rough Surfaces}

We shall consider the scattering of an acoustic or electromagnetic time-harmonic plane wave

$$
\begin{aligned}
\widetilde{v}_{\text {inc }}(x, y, t) & =\exp (-i \omega t) v_{\text {inc }}(x, y) \\
& =\exp (-i \omega t) \exp (i \alpha x-i \beta y)
\end{aligned}
$$

incident upon a rough $d$-periodic surface

$$
y=g(x), \quad g(x+d)=g(x),
$$

and we shall be interested in predictions of the generated scattered field

$$
\widetilde{v}_{\text {scat }}(x, y, t)=\exp (-i \omega t) v_{\text {scat }}(x, y) .
$$

Here, of course, $\widetilde{v}$ denotes the pressure in acoustics or the component of the electric or magnetic field parallel to the invariant $(z)$ direction in electromagnetics. In any case, the (reduced) scattered field $v_{\text {scat }}(x, y)$ propagates according to the Helmholtz equation

$$
\Delta v_{\text {scat }}+k^{2} v_{\text {scat }}=0
$$

on $y>g(x)$, where the wave number $k$ satisfies $k^{2}=\alpha^{2}$ $+\beta^{2}=(2 \pi / \lambda)^{2}$ and $\lambda$ is the wavelength of radiation. The uniqueness of solutions coupled to the periodicity of the profile $g(x)$ then implies that $v_{\text {scat }}$ will be "quasiperiodic" in $x$, that is,

$$
v_{\text {scat }}(x+d, y)=\exp (i \alpha d) v_{\text {scat }}(x, y) .
$$

Any of a number of physically relevant boundary conditions can be imposed on the rough surface. For the sake of definiteness, we shall work with a pressure release or perfectly conducting (TE) surface, where

$$
v_{\text {scat }}(x, g(x))=-v_{\text {inc }}(x, g(x))=-\exp [i \alpha x-i \beta g(x)] .
$$

Finally, the Sommerfeld radiation condition must be imposed to specify the physical solution. Within the present context, this condition can be stated in terms of the Rayleigh series: If $a$ is any number satisfying $a$ $>\max |g(x)|$, then the scattered field in $y>a$ must admit a representation

$$
v_{\text {scat }}(x, y)=\sum_{p=-\infty}^{\infty} B_{p} \exp \left(i \alpha_{p} x+i \beta_{p} y\right)
$$

as a superposition of outgoing waves. Here

$$
\alpha_{p}=\alpha+\frac{2 \pi}{d} p, \quad \beta_{p}=\sqrt{k^{2}-\alpha_{p}^{2}} .
$$

Equivalently, the radiation condition can be stated in terms of a "Dirichlet-to-Neumann" operator (DNO), with the advantage that the resulting problem is formulated in a bounded domain. We recall that given a surface $y$ $=\sigma(x)$ the DNO $T(\sigma)$ associated with the scattering problem (2.1) and (2.2) on $y>\sigma(x)$ is an operator that acts on (quasi-periodic) functions $\xi(x)$ defined on $y$ $=\sigma(x)$ : The operator maps the function $\xi$ onto a function $T(\sigma)[\xi]$, also defined on the surface, according to

$$
T(\sigma)[\xi](x)=\frac{\partial v_{\text {scat }}}{\partial n(x)}(x, \sigma(x)),
$$

where $v_{\text {scat }}$ is the (quasi-periodic) solution to Eq. (2.1) with boundary condition

$$
v_{\text {scat }}(x, \sigma(x))=\xi(x) .
$$

In particular, if $\sigma(x) \equiv a>\max |g(x)|$ and

$$
\xi(x)=\sum_{p=-\infty}^{\infty} \hat{\xi}_{p} \exp \left(i \alpha_{p} x\right),
$$

the corresponding solution in $y>a$ is

$$
v_{\text {scat }}(x, y)=\sum_{p=-\infty}^{\infty} \hat{\xi}_{p} \exp \left[i \alpha_{p} x+i \beta_{p}(y-a)\right],
$$

so that

$$
\begin{aligned}
T(\sigma)[\xi](x) & =T(a)[\xi](x)=-\frac{\partial v_{\text {scat }}}{\partial y}(x, a) \\
& =\sum_{p=-\infty}^{\infty}\left(-i \beta_{p}\right) \hat{\xi}_{p} \exp \left(i \alpha_{p} x\right) .
\end{aligned}
$$


Hence an equivalent form of Sommerfeld's condition is given by

$$
\frac{\partial v_{\text {scat }}}{\partial y}(x, a)+T(a)\left[v_{\text {scat }}(\cdot, a)\right](x)=0 .
$$

Summarizing, and denoting $v_{\text {scat }}(x, y)$ by $v(x, y)$, we shall seek a solution of the system of equations

$$
\begin{aligned}
& \Delta v+k^{2} v=0, \\
& v(x, g(x))=-\exp [i \alpha x-i \beta g(x)], \\
& \partial_{y} v(x, a)+T(a)[v(\cdot, a)](x)=0, \\
& v(x+d, y)=\exp (i \alpha d) v(x, y)
\end{aligned}
$$

for $g(x)<y<a$.

\section{B. Field Expansions}

The basic idea behind shape-perturbation methods is to exploit the explicit solvability of Eqs. (2.5) in the case where $g(x) \equiv 0$, that is, for a flat surface. In this case, the law of reflection gives, for $y>0$,

$$
v=v_{0}(x, y)=-\exp (i \alpha x+i \beta y),
$$

and, more generally, if $v_{\text {inc }}(x, 0)=-d_{p} \exp \left(i \alpha_{p} x\right)$, then

$$
v(x, y)=d_{p} \exp \left(i \alpha_{p} x+i \beta_{p} y\right) .
$$

These relations can be used to advantage if a general surface $y=g(x)$ is embedded in a family $y=\delta g(x), 0 \leqslant \delta$ $\leqslant 1$, and the solutions $V(x, y ; \delta)$ of Eqs. (2.5), with $g(x)$ replaced by $\delta g(x)$, are sought in the form of a perturbation series:

$$
V(x, y ; \delta)=\sum_{n=0}^{\infty} v_{n}(x, y) \delta^{n}
$$

Indeed, as can be readily checked, the functions $v_{n}$ are, at least formally, solutions to scattering problems over a flat interface and can therefore be explicitly found. More precisely, the functions $v_{n}$ satisfy (see, e.g., Ref. 24)

$$
\begin{aligned}
& \Delta v_{n}+k^{2} v_{n}=0, \\
& v_{n}(x, 0)=P_{n}(x), \\
& \partial_{y} v_{n}(x, a)+T(a)\left[v_{n}(\cdot, a)\right](x)=0, \\
& v_{n}(x+d, y)=\exp (i \alpha d) v_{n}(x, y) .
\end{aligned}
$$

for $0<y<a$. To find the specific form of the "incident field" $P_{n}(x)$, one resorts to the condition satisfied by $V(x, y ; \delta)$ at $y=\delta g(x)$, namely,

$$
V(x, \delta g(x) ; \delta)=-\exp [i \alpha x-i \beta \delta(x)] .
$$

Formal differentiations with respect to $\delta$ then yield

$$
\begin{aligned}
P_{n}(x)= & -\exp (i \alpha x) \frac{[-i \beta g(x)]^{n}}{n !} \\
& -\sum_{l=0}^{n-1} \frac{[g(x)]^{n-l}}{(n-l) !} \partial_{y}^{n-l} v_{l}(x, 0),
\end{aligned}
$$

so that the equations can be solved recursively, starting from Eq. (2.6), to define

$$
v(x, y)=V(x, y ; \delta=1)=\sum_{n=0}^{\infty} v_{n}(x, y)
$$

This formal procedure (or slight variations thereof) has been used extensively in both low- and high-order implementations. It has been referred to by a variety of names, including small-perturbation method, ${ }^{38,39}$ Rayleigh-Fourier Method, ${ }^{17}$ Rayleigh-Rice theory, ${ }^{16}$ iterative series solution, ${ }^{15}$ etc. Here we shall refer to it with the generic name of method of "field expansion" (FE). Our choice for this name is motivated by the actual expansion of fields that underlies the method; this name will also help us distinguish it from the operator expansion (OE) method, which we review in Subsection 2.C.

As we mentioned in Section 1, the convergence of the series in Eq. (2.11) was a subject of much controversy over the last 50 years and was eventually resolved in Ref. 23. There it was also shown that, for analytic profiles $g(x)$, the function (scattered field) $V(x, y ; \delta)$ is analytic for all values of $\delta$ on the real line. Besides its intrinsic theoretical value, this observation has a significant practical consequence, as it guarantees that the Taylor coefficients $v_{n}(x, y)$ contain all the necessary information to determine the values of $V(x, y ; \delta)$ for every $\delta$, even beyond the disk of convergence of the series. As proposed in Ref. 23, the FE approach can be combined with mechanisms of analytic continuation (e.g., conformal mappings, ${ }^{24}$ Padé approximation, ${ }^{25,26,29}$ etc.) to produce a method that can accurately compute scattering returns for large deformations of a plane that lie well beyond the radius of convergence of the perturbation series.

For a practical implementation of this approach, it is convenient to work in Fourier space. More precisely, the most general solution to Eqs. (2.8a), (2.8c), and (2.8d) is of the form

$$
v_{n}(x, y)=\sum_{p=-\infty}^{\infty} d_{n, p} \exp \left(i \alpha_{p} x+i \beta_{p} y\right)
$$

for some coefficients $d_{n, p}$. Equations (2.8b) and (2.10) then translate into a recursion for these coefficients ${ }^{24}$ :

$$
d_{n, p}=-(-i \beta)^{n} C_{n, p}-\sum_{l=0}^{n-1} \sum_{q=-\infty}^{\infty} C_{n-l, p-q}\left(i \beta_{q}\right)^{n-l} d_{l, q}
$$

where

$$
[g(x)]^{l} / l !=\sum_{p=-\infty}^{\infty} C_{l, p} \exp (i p x) .
$$

Note that the inner sum in Eq. (2.13) is a discrete convolution, so that it can be performed efficiently by means of fast Fourier transforms (FFTs). Equivalently, an efficient implementation of Eqs. (2.8b) and (2.10) can be attained by performing all multiplications [e.g., by powers of the profile $g(x)]$ in physical space and all differentiations in the frequency domain and by using FFTs to transform from one to the other. Also, from Eqs. (2.3), (2.11), and (2.12), we have 


$$
B_{p}=\sum_{n=0}^{\infty} d_{n, p}
$$

so that the Rayleigh amplitudes can be readily computed once Eq. (2.13) has been resolved. In fact, as we said, the series in Eq. (2.15) may not converge for large perturbations. As has been shown, however, Padé approximation provides an effective analytic continuation mechanism in this case (see, e.g., Ref. 29).

\section{Operator Expansions}

An alternative and elegant shape-perturbation scheme was pioneered by Milder et al. ${ }^{30-36,38}$ in scattering calculations, based on earlier work on simulations of the evolution of gravity water waves ${ }^{40-42}$ (see also Refs. 43 and 44). In the context of two-dimensional, periodic scattering configurations, this approach starts with consideration of the Helmholtz integral for the scattered field in $y>g(x)$ :

$$
\begin{aligned}
v(x, y)= & \int_{0}^{d}\left[\frac{\partial \Phi}{\partial n\left(x^{\prime}\right)}\left(x-x^{\prime}, y-g\left(x^{\prime}\right)\right) v\left(x^{\prime}, g\left(x^{\prime}\right)\right)\right. \\
& -\frac{\partial v}{\partial n\left(x^{\prime}\right)}\left(x^{\prime}, g\left(x^{\prime}\right)\right) \\
& \left.\times \Phi\left(x-x^{\prime}, y-g\left(x^{\prime}\right)\right)\right] \mathrm{d} x^{\prime}
\end{aligned}
$$

where $^{23}$

$$
\begin{aligned}
\Phi(x, y)= & \frac{i}{4} \sum_{n=-\infty}^{\infty} \exp (-i \alpha n d) H_{0}^{(1)}\left(k \left[(x+n d)^{2}\right.\right. \\
& \left.\left.+y^{2}\right]^{1 / 2}\right) \\
= & \frac{i}{2 d} \sum_{p=-\infty}^{\infty} \frac{\exp \left(i \beta_{p}|y|\right)}{\beta_{p}} \exp \left(i \alpha_{p} x\right)
\end{aligned}
$$

is the (periodized) free-space Green's function and $n(x)$ $=\left(\partial_{x} g(x),-1\right)$ is the (unnormalized) outward normal vector to the scattering surface. Next, we introduce the Dirichlet-to-Neumann operator (DNO) $T(g)$, as defined in Subsection 2.A, in terms of which Eq. (2.16) can be written in the form

$$
\begin{aligned}
v(x, y)= & \int_{0}^{d}\left[-\frac{\partial \Phi}{\partial n\left(x^{\prime}\right)}\left(x-x^{\prime}, y-g\left(x^{\prime}\right)\right) f_{\mathrm{inc}}\left(x^{\prime}\right)\right. \\
& \left.+\Phi\left(x-x^{\prime}, y-g\left(x^{\prime}\right)\right) T(g)\left[f_{\mathrm{inc}}\right]\left(x^{\prime}\right)\right] \mathrm{d} x^{\prime}
\end{aligned}
$$

where we have used $v(x, g(x))=-v_{\text {inc }}(x, g(x))$ and have set

$$
f_{\text {inc }}(x)=v_{\text {inc }}(x, g(x))=\exp [i \alpha x-i \beta g(x)] \text {. }
$$

Substituting the modal expansion of the Green's function (2.17) into Eq. (2.18), we obtain, for $y>\max |g(x)|$,

$$
v(x, y)=\sum_{p=-\infty}^{\infty} B_{p} \exp \left(i \alpha_{p} x+i \beta_{p} y\right)
$$

where

$$
\begin{aligned}
B_{p}= & \frac{i}{2 d \beta_{p}} \int_{0}^{d} f_{p}\left(x^{\prime}\right)\left\{\left[i \alpha_{p} \partial_{x} g\left(x^{\prime}\right)-i \beta_{p}\right]\right. \\
& +T(g)\}\left[f_{\text {inc }}\right]\left(x^{\prime}\right) \mathrm{d} x^{\prime}, \\
f_{p}(x)= & \exp \left[-i \alpha_{p} x-i \beta_{p} g(x)\right] .
\end{aligned}
$$

As follows from Eq. (2.20), a central component of the method relates to the calculation of the DNO. For this, a perturbative scheme appears to be particularly appropriate, as we recall (cf. Subsection 2.A) that the operator can be explicitly found for a flat interface. Indeed, letting $a$ $=0$ in Eq. (2.4), we have

$$
T(0)\left\{\sum_{p=-\infty}^{\infty} \hat{\xi}_{p} \exp \left(i \alpha_{p} x\right)\right\}=\sum_{p=-\infty}^{\infty}\left(-i \beta_{p}\right) \hat{\xi}_{p} \exp \left(i \alpha_{p} x\right) .
$$

For a general surface $y=g(x)$, we shall seek an expression for the operator $T(g)$ in the form of a perturbation series:

$$
T(g)=\left.T(\delta g)\right|_{\delta=1}=\left.\left[\sum_{n=0}^{\infty} T_{n}(g) \delta^{n}\right]\right|_{\delta=1} .
$$

To find a recursion for the (operator) coefficients $T_{n}(g)$, we note that the functions

$$
w_{p}(x, y)=\exp \left(i \alpha_{p} x+i \beta_{p} y\right)
$$

solve Eqs. (2.5a), (2.5c), and (2.5d), so that

$$
\begin{aligned}
& T(\delta g) {\left[\exp \left\{i \alpha_{p} x+i \beta_{p} \delta g(x)\right\}\right] } \\
&=\left[i \alpha_{p} \delta \partial_{x} g(x)-i \beta_{p}\right] \exp \left[i \alpha_{p} x+i \beta_{p} \delta g(x)\right] .
\end{aligned}
$$

Expanding Eq. (2.24) in powers of $\delta$ and equating like powers, we obtain

$T_{n}(g)\left[\exp \left(i \alpha_{p} x\right)\right]$

$$
\begin{aligned}
= & {\left[\partial_{x} g(x)\right]\left(i \alpha_{p}\right)\left(i \beta_{p}\right)^{n-1} \frac{[g(x)]^{n-1}}{(n-1) !} \exp \left(i \alpha_{p} x\right) } \\
& -\left(i \beta_{p}\right)^{n+1} \frac{[g(x)]^{n}}{n !} \exp \left(i \alpha_{p} x\right) \\
& -\sum_{l=0}^{n-1} T_{l}(g)\left[\left(i \beta_{p}\right)^{n-l} \frac{\{g(x)\}^{n-l}}{(n-l) !} \exp \left(i \alpha_{p} x\right)\right] .
\end{aligned}
$$

Therefore, if

$$
\xi(x)=\sum_{p=-\infty}^{\infty} \hat{\xi}_{p} \exp \left(i \alpha_{p} x\right)
$$

then, using the linear character of the operator $T_{n}(g)$, we find that 


$$
\begin{aligned}
T_{n}(g)[\xi]= & \left.\partial_{x} g(x)\right] \frac{[g(x)]^{n-1}}{(n-1) !} \sum_{p=-\infty}^{\infty}\left(i \alpha_{p}\right) \\
& \times\left(i \beta_{p}\right)^{n-1} \hat{\xi}_{p} \exp \left(i \alpha_{p} x\right) \\
& -\frac{[g(x)]^{n}}{n !} \sum_{p=-\infty}^{\infty}\left(i \beta_{p}\right)^{n+1} \hat{\xi}_{p} \exp \left(i \alpha_{p} x\right) \\
& -\sum_{l=0}^{n-1} T_{l}(g)\left[\frac{\{g(x)\}^{n-l}}{(n-l) !}\right. \\
& \left.\times \sum_{p=-\infty}^{\infty}\left(i \beta_{p}\right)^{n-l} \hat{\xi}_{p} \exp \left(i \alpha_{p} x\right)\right]
\end{aligned}
$$

or, equivalently, using $\beta_{p}^{2}=k^{2}-\alpha_{p}^{2}$,

$$
\begin{aligned}
T_{n}(g)[\xi]= & \partial_{x}\left\{\frac{[g(x)]^{n}}{n !} \sum_{p=-\infty}^{\infty}\left(i \alpha_{p}\right)\left(i \beta_{p}\right)^{n-1} \hat{\xi}_{p} \exp \left(i \alpha_{p} x\right)\right\} \\
& +k^{2} \frac{[g(x)]^{n}}{n !} \sum_{p=-\infty}^{\infty}\left(i \beta_{p}\right)^{n-1} \hat{\xi}_{p} \exp \left(i \alpha_{p} x\right) \\
& -\sum_{l=0}^{n-1} T_{l}(g)\left[\frac{\{g(x)\}^{n-l}}{(n-l) !}\right. \\
& \left.\times \sum_{p=-\infty}^{\infty}\left(i \beta_{p}\right)^{n-l} \hat{\xi}_{p} \exp \left(i \alpha_{p} x\right)\right] .
\end{aligned}
$$

Setting

$$
D=-i \partial_{x}
$$

and introducing a (pseudo-differential) operator $\beta_{D}$, defined by

$$
\beta_{D}[\xi]=\sum_{p=-\infty}^{\infty} \beta_{p} \hat{\xi}_{p} \exp \left(i \alpha_{p} x\right),
$$

we can write Eq. (2.25) as

$$
\begin{aligned}
T_{n}(g)[\xi]= & \partial_{x}\left\{\frac{[g(x)]^{n}}{n !}\left(i \beta_{D}\right)^{n-1} \partial_{x} \xi\right\} \\
& +k^{2} \frac{[g(x)]^{n}}{n !}\left(i \beta_{D}\right)^{n-1} \xi \\
& -\sum_{l=0}^{n-1} T_{l}(g)\left[\frac{\{g(x)\}^{n-l}}{(n-l) !}\left(i \beta_{D}\right)^{n-1} \xi\right] .
\end{aligned}
$$

A further simplification is possible at this point, as first noted in Refs. 45 and 46 . Indeed, note that Eq. (2.28) for $T_{n}(g)$ involves the operators $T_{l}(g), 0 \leqslant l \leqslant n-1$, evaluated on functions that change with the order $n$. In contrast, using the self-adjointness of these operators (and the fact that the operator $\beta_{D}$ commutes with differentiation), we can rewrite Eq. (2.28) in the form

$$
\begin{aligned}
T_{n}(g)[\xi]= & \left(i \beta_{D}\right)^{n-1}\left\{\partial_{x} \frac{[g(x)]^{n}}{n !} \partial_{x} \xi+k^{2} \frac{[g(x)]^{n}}{n !} \xi\right\} \\
& -\sum_{l=0}^{n-1}\left(i \beta_{D}\right)^{n-l} \frac{[g(x)]^{n-l}}{(n-l) !} T_{l}(g)[\xi] .
\end{aligned}
$$

This latter form significantly reduces the computational effort, as now the operators $T_{l}(g), 0 \leqslant l \leqslant n-1$, are always evaluated on the same function $\xi$, independently of $n$, and can therefore be stored and reused at each order. In fact, with this addition, a numerical implementation of this approach can be attained with prescriptions similar to those described above in the context of the FE procedure. Indeed, here the values of $T_{l}(g)[\xi]$ on a fixed equispaced grid can, once again, be recursively obtained from Eq. (2.29) by effecting multiplications in physical space and applications of $\beta_{D}$ in the frequency domain and by alternating between these by means of FFTs.

\section{Partial Field Expansions}

When one is interested in the scatter off a specific surface $y=g(x)$ [and not on that generated by the intermediate surfaces $y=\delta g(x)$ ], a particularly appealing feature of the OE procedure is that only the DNO is approximated perturbatively. In particular, the function $f_{\text {inc }}$ in Eq. (2.19) is not expanded in powers of $g$ [and neither is $f_{p}$ in Eq. (2.21)]. This suggests an alternative to the FE scheme presented in Subsection 2.B, wherein the incoming wave is not expanded in powers of $\delta$. More precisely, an alternative form of the recursions can be derived if we replace Eq. (2.9) by

$$
V(x, \delta g(x) ; \delta)=-\exp [i \alpha x-i \beta g(x)],
$$

so that, at zero order, we have

$$
v_{0}(x, 0)=-\exp [i \alpha x-i \beta g(x)] .
$$

Indeed, in this case, Eqs. (2.8) are replaced by a similar recursion, but now $P_{n}$ in Eq. (2.10) takes on the simpler form

$$
P_{n}(x)=-\sum_{l=0}^{n-1} \frac{[g(x)]^{n-l}}{(n-l) !} \partial_{y}^{n-l} v_{l}(x, 0) .
$$

As expected, this modified approach, which we shall refer to as a partial field expansion (PFE), generally exhibits better convergence properties than the FE method; see Fig. 1.

As follows from Eqs. (2.13), (2.29), and (2.31) and as explained above, methods based on shape deformations lead, in this case, to very simple and efficient algorithms, entailing only straightforward FFTs and multiplications. In fact, it is these characteristics that can make these approaches the method of choice over other classical procedures (e.g., integral equations, finite elements, etc.) in many instances; see, e.g., Refs. 47 and 48 for a recent example of application and comparison with alternative methods.

In fact, the advantages of shape-deformation methods over other rigorous numerical schemes can become more pronounced for scalar and vector problems in three dimensions. Indeed, in this case, finite-element approaches, for instance, must deal with volumetric discreti- 


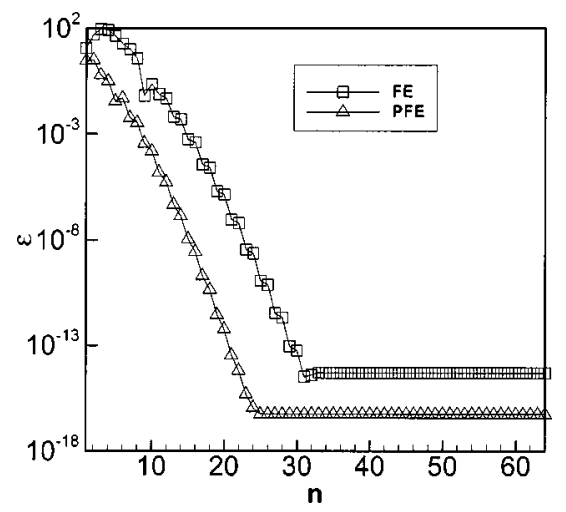

Fig. 1. Plot of "energy defect" (error in conservation of energy) versus perturbation order $n$ for a sinusoidal profile under normal incidence; the height-to-period ratio is 0.05 , and the wavelengthto-period ratio is 0.065. A comparison is given of $\mathrm{FE}$ and $\mathrm{PFE}$ implementations.

zations and artificial boundary conditions, ${ }^{49}$ while the design of efficient integral equation methods faces significant challenges in the design of both appropriate quadrature rules and efficient matrix inversion mechanisms; see, e.g., Refs. 39 and 50. In contrast, the FE and PFE approaches described above can be readily extended to these cases, leading to similarly simple recurrences. ${ }^{26,28,48}$ The $\mathrm{OE}$ approach can also be extended, although, for general three-dimensional electromagnetic problems, it requires a more careful treatment on account of the more complicated boundary conditions that must be enforced in this case and that lead to a correspondingly more complicated definition for the relevant DNO. ${ }^{36,37}$ [In this connection, we point out that the treatment in Refs. 36 and 37 does not constitute the only possibility, and, in fact, a perhaps more natural version of the recurrence can be derived. Indeed, for instance in the case of perfectly conducting surfaces, such a recurrence can be derived by defining the DNO as directly providing the surface current from knowledge of the electric field on the surface for use in a Stratton-Chu formula ${ }^{51}$ - the analog of Eq. (2.16). The recurrence for the perturbation coefficients of the DNO can then be derived by simply enforcing that the operator vanish on normal fields and that it satisfy the analog of Eq. (2.24) on electric fields that are tangential to the surface.]

The aforementioned advantages of shape-deformation methods hold throughout their domain of applicability. And, as was shown in Refs. 23 and 52, the theoretical validity of boundary perturbation expansions extends to perturbations of arbitrary order and arbitrary size. However, a numerical implementation of these schemes leads to a restricted domain of applicability on account of inaccuracies that arise from finite arithmetic and roundoff errors, which have, until now, remained unexplained. The origins of these instabilities are elucidated in Section 3 in the context of both the $\mathrm{OE}$ and FE implementations.

\section{CANCELLATIONS AND CONDITIONING}

It is important to note that the derivations of the recursions in Section 2 are formal in nature. Indeed, as we mentioned, the question of convergence of shape- perturbation expansions has a long history, as does the question of validity of recursions such as those in Eqs. (2.8) and (2.29). With regard to the issue of convergence, a rather general result can be derived from the work of Calderón, ${ }^{53}$ and Coifman and Meyer ${ }^{54}$ on the theory of singular integrals. Indeed, it follows from this work that the series in Eq. (2.22) converges for sufficiently small $\delta$ (in appropriate functional spaces) provided that $g(x)$ is a Lipschitz-continuous function [i.e., there is a constant $M$ such that $\left|g(x)-g\left(x^{\prime}\right)\right| \leqslant M\left|x-x^{\prime}\right|$ for $0 \leqslant x, x^{\prime}$ $\leqslant d]$. From this, in turn, it also follows that the series (2.7) converges inside the domain $y>\delta g(x)$. However, these results do not shed light on the, perhaps more important, issue of validity of the recursive formulas. This, in fact, is a more subtle issue, as it involves the behavior of the fields on the boundary $y=\delta g(x)$ of their domains of definition. An affirmative answer on the validity of the recursive relations was finally provided in Ref. 23 for the case where $g(x)$ is an analytic function.

The discrepancy in the smoothness requirements of the results quoted above, Lipschitz regularity for Ref. 54 and analyticity for Ref. 23 , can be used to motivate one of our main observations. Indeed, the analyticity assumption in Ref. 23 was fundamental to the derivation of the recurrence (2.8), which, when analyzed in detail, can be shown to involve derivatives of $g(x)$ of arbitrarily high order. This property is shared by the recurrence (2.29), where it is, in fact, much more obvious ( $\beta_{D}$ is a pseudo-differential operator of order 1; i.e., with regard to regularity properties, it acts like differentiation). On the other hand, as we said, the results in Ref. 54 apply to general "rough" (Lipschitz) perturbations of a plane. As we have shown, ${ }^{55,56}$ this apparent contradiction is at the heart of the instabilities that may arise in $\mathrm{OE}$ and FE calculations. Indeed, as argued there, substantial cancellations occur in Eqs. (2.8b) and (2.29), so that the overall sums on their respective right-hand sides give rise to finite quantities in spite of possible singularities in the individual terms.

In the remainder of this section, we shall substantiate this argument with some analytical remarks and some numerical examples. In particular, we shall first explain the manner in which the cancellations manifest themselves at low orders, where explicit calculations can be performed to reveal them. In addition to providing a direct verification of their presence, these low-order calculations will also shed light on the rather dramatic effect of cancellations in high-order simulations (Subsection 3.C). In Section 4, we shall further demonstrate how these cancellations preclude an iterative estimation of the functions $v_{n}$ in Eqs. (2.8) or $T_{n}(g)[\xi]$ in Eq. (2.29), as any use of the triangle inequality destroys these, rendering a useless bound. Interestingly, we will also show that a simple change of independent variables can be made to implicitly account for cancellations, allowing then for an iterative estimation in the new coordinates.

\section{A. Cancellations in the Operator Expansion Method} In the $\mathrm{OE}$ formulation, a cancellation of high-order derivatives occurs at every order and can, in fact, be explicitly identified at low orders in Eq. (2.29). Indeed, for $n$ $=0,1,2$, Eq. (2.29) reads as 


$$
\begin{aligned}
T_{0}[\xi]= & -i \beta_{D} \xi \\
T_{1}(g)[\xi]= & -D g D \xi+k^{2} g \xi-i \beta_{D} g T_{0}[\xi], \\
T_{2}(g)[\xi]= & i \beta_{D}\left\{-D\left(g^{2} / 2\right) D \xi+k^{2}\left(g^{2} / 2\right) \xi\right. \\
& \left.-i \beta_{D}\left(g^{2} / 2\right) T_{0}[\xi]-g T_{1}(g)[\xi]\right\},
\end{aligned}
$$

where the $D$ and the $\beta_{D}$ are defined as in Eqs. (2.26) and (2.27), respectively. Note that $T_{1}(g)[\xi]$ appears to involve second derivatives of the data $\xi$ and that $T_{2}(g)[\xi]$ appears to involve third derivatives. On the other hand (see Appendix A, Theorem A.1), if $f$ is Lipschitz continuous and $\eta$ is square-integrable, the operator $\beta_{D}$ satisfies a kind of "product rule:"

$$
\beta_{D}[f \eta]-f \beta_{D}[\eta]=R_{f}[\eta],
$$

where $R_{f}[\eta]$ is more regular than either of the two terms on the left-hand side (in particular, it is itself squareintegrable). In other words,

$$
\text { most singular part of } \beta_{D}[f \eta]=f \beta_{D}[\eta] \text {. }
$$

Thus we can write

$$
\begin{aligned}
T_{1}(g)[\xi]= & -(D g)(D \xi)-g\left(D^{2} \xi\right)+k^{2} g \xi-g \beta_{D}^{2} \xi \\
& -R_{g}\left[\beta_{D} \xi\right],
\end{aligned}
$$

and since

$$
\beta_{D}^{2}=k^{2}-D^{2},
$$

we see that the second derivatives of $\xi$ and the term involving $k^{2}$ exactly cancel out, yielding

$$
T_{1}(g)[\xi]=-(D g)(D \xi)-R_{g}\left[\beta_{D} \xi\right] .
$$

Similarly, using Eq. (3.4) in Eq. (3.1c) and expanding the derivatives, we get

$$
\begin{aligned}
T_{2}(g)[\xi]= & i \beta_{D}\left\{-\left(g^{2} / 2\right) D^{2} \xi-g(D g)(D \xi)+k^{2}\left(g^{2} / 2\right) \xi\right. \\
& -\left(g^{2} / 2\right) \beta_{D}^{2} \xi-R_{g^{2} / 2}\left[\beta_{D} \xi\right]-g((D g)(D \xi) \\
& \left.\left.-R_{g}\left[\beta_{D} \xi\right]\right)\right\}
\end{aligned}
$$

or, deleting terms that exactly cancel out (first/third/ fourth and second/sixth terms on the right-hand side),

$$
T_{2}(g)[\xi]=i \beta_{D} S_{g}\left[\beta_{D} \xi\right],
$$

where the operator $S_{f}$ is defined as

$$
S_{f}[\eta]=-R_{f^{2} / 2}[\eta]+f R_{f}[\eta] .
$$

Again here, and in contrast with the individual terms on the right-hand side of Eq. (3.1c), the right-hand side of Eq. (3.5) is square-integrable, as the operator $S_{f}$ in Eq. (3.6) maps square-integrable functions onto functions with a square-integrable derivative (see Appendix A, Theorem A.2).

\section{B. Cancellations in Field Expansion Methods}

Not surprisingly, the cancellations in the FE and PFE approaches lead to consideration of the same operators as those in Subsection 3.A. The behavior of the "cancelled equations" in this case, however, is somewhat different from that of the corresponding OE relations [cf. Eqs. (3.4) and (3.5)], as we explain next.

As discussed in Subsections 2.B and 2.D, the recurrences for the $\mathrm{FE}$ and $\mathrm{PFE}$ approaches are similar in nature and differ only in the manner in which the incident wave is treated. For this reason, cancellations in these procedures arise in precisely the same way, which, for the sake of brevity, we shall exemplify on the PFE approach; as will be clear from these developments, entirely analogous remarks apply to the FE scheme.

To shed light on the cancellations in the PFE method, we begin by noting that the partial derivatives with respect to the transverse $(y)$ variable in Eq. (2.31) are closely related to the operator $\beta_{D}$ in Eq. (2.27); indeed, we have

$$
\partial_{y}=i \beta_{D} \text {. }
$$

For $n=0,1,2$ then, and letting $\xi$ denote the incident wave on the scattering surface, we can write the PFE recurrences as

$$
\begin{aligned}
& v_{0}=\xi, \\
& v_{1}=-i g \beta_{D} v_{0}, \\
& v_{2}=\frac{g^{2}}{2} \beta_{D}^{2} v_{0}-i g \beta_{D} v_{1} .
\end{aligned}
$$

If $\xi$ has a square-integrable derivative, it follows from Eqs. (3.7) that both $v_{0}$ and $v_{1}$ are well defined. To see how cancellations arise in $v_{2}$, we write, from Eq. (3.7c),

$$
v_{2}=\frac{g^{2}}{2}\left(k^{2}-D^{2}\right) \xi-g \beta_{D} g \beta_{D} \xi,
$$

where we have used Eqs. (3.7a) and (3.7b) and the equality (3.3). Then, from Eq. (3.2),

$$
\begin{aligned}
v_{2} & =\frac{g^{2}}{2}\left(k^{2}-D^{2}\right) \xi-g^{2} \beta_{D}^{2} \xi-g R_{g}\left[\beta_{D} \xi\right] \\
& =\frac{g^{2}}{2}\left(k^{2}-D^{2}\right) \xi-g^{2}\left(k^{2}-D^{2}\right) \xi-g R_{g}\left[\beta_{D} \xi\right] \\
& =-\frac{g^{2}}{2}\left(k^{2}-D^{2}\right) \xi-g R_{g}\left[\beta_{D} \xi\right] .
\end{aligned}
$$

On the other hand, we also have that

$$
\beta_{D} \frac{g^{2}}{2} \beta_{D} \xi=\frac{g^{2}}{2}\left(k^{2}-D^{2}\right) \xi+R_{g^{2} / 2}\left[\beta_{D} \xi\right],
$$

so that Eq. (3.9) can be transformed to

$$
v_{2}=-\beta_{D}\left[\frac{g^{2}}{2} \beta_{D} \xi\right]+R_{g^{2} / 2}\left[\beta_{D} \xi\right]-g R_{g}\left[\beta_{D} \xi\right]
$$

or, by using Eq. (3.6),

$$
v_{2}=-\beta_{D}\left[\frac{g^{2}}{2} \beta_{D} \xi\right]-S_{g}\left[\beta_{D} \xi\right] .
$$

As follows from the above derivation, this last form of definition for $v_{2}$ rectifies some cancellations present in the original form $(3.7 \mathrm{c})$. Indeed, from Theorem A.2, the second term in Eq. (3.11) is more regular than any of the terms in Eq. (3.7c). The first term in Eq. (3.11), on the 
other hand, precisely identifies the most singular part of $v_{2}$ and expresses it in a manner that is better suited for approximation. Indeed, a comparative analysis of this term with the first term in Eq. (3.7c), for instance, reveals that the latter will lead to larger errors on truncation of the profile $g$ and the incidence $\xi$. To illustrate this additional and subtle effect, we let $b_{r}$ and $C_{2, r}$ [cf. Eq. (2.14)] denote the Fourier coefficients of $\beta_{D} \xi$ and $[g(x)]^{2} / 2$, respectively:

$$
\begin{aligned}
\beta_{D} \xi(x) & =\sum_{r=-\infty}^{\infty} b_{r} \exp \left(i \alpha_{p} x\right) \\
{[g(x)]^{2} / 2 } & =\sum_{r=-\infty}^{\infty} C_{2, r} \exp [i(2 \pi / d) p x] .
\end{aligned}
$$

Then the first term in Eq. (3.7c) is

$$
\frac{[g(x)]^{2}}{2} \beta_{D}^{2} \xi(x)=\sum_{r=-\infty}^{\infty}\left(\sum_{p=-\infty}^{\infty} C_{2, r-p} \beta_{p} b_{p}\right) \exp \left(i \alpha_{r} x\right)
$$

while that in Eq. (3.11) reads as

$$
\beta_{D}\left[\frac{g^{2}}{2} \beta_{D} \xi\right](x)=\sum_{r=-\infty}^{\infty} \beta_{r}\left(\sum_{p=-\infty}^{\infty} C_{2, r-p} b_{p}\right) \exp \left(i \alpha_{r} x\right) .
$$

The issue here then is the approximation of the Fourier coefficients in Eqs. (3.12) and (3.13) with the use of truncated series, that is, as

$$
\begin{aligned}
& \sum_{p=-F}^{F} C_{2, r-p} \beta_{p} b_{p}, \\
& \beta_{r} \sum_{p=-F}^{F} C_{2, r-p} b_{p},
\end{aligned}
$$

respectively. The benefits of the formulation (3.11) are now evident from Eqs. (3.14) and (3.15) as the additional factor $\beta_{p}(\approx|p|)$ inside the sum of the former decreases the rate of convergence of the series and translates into larger errors. In fact, these effects are clearly influenced by the regularity, or lack thereof, of both the profile $g$ and the incidence $\xi$, as it is these characteristics that determine the decay of the coefficients $C_{2, r}$ and $b_{r}$ (and thus the speed of convergence of the series). Moreover, even in the case of smooth data, the detrimental effects of these cancellations are consistently accentuated at higher orders. Indeed, proceeding as in the derivation of Eq. (3.11), we also find that, after significant cancellation,

$$
v_{3}=i \frac{g^{3}}{6} \beta_{D}^{3} v_{0}+\frac{g^{2}}{2} \beta_{D}^{2} v_{1}-i g \beta_{D} v_{2}
$$

can be reduced to

$$
v_{3}(x)=i \beta_{D}^{2}\left[\frac{g^{3}}{6} \beta_{D} \xi\right]+i g \beta_{D} S_{g}\left[\beta_{D} \xi\right],
$$

where again the first term entails the most singular part, on account of Theorem A.2; in fact, it can be shown inductively that most singular part of $v_{n}=(-i)^{n} \beta_{D}^{n-1}\left[\frac{g^{n}}{n !} \beta_{D} \xi\right]$.

From Eq. (3.17) and letting $C_{3, r}$ denote the Fourier coefficients of $g^{3} / 6$, we see that the analog of the series (3.14) at third order, namely a truncated version of the first term in Eq. (3.16), leads to

$$
i \sum_{p=-F}^{F} C_{3, r-p} \beta_{p}^{2} b_{p}
$$

while that corresponding to the first term on the righthand side of Eq. (3.17) is

$$
i \beta_{r}^{2} \sum_{p=-F}^{F} C_{3, r-p} b_{p}
$$

These latter expressions then imply that the relative error in an approximation of the original recursion significantly deteriorates at third order, while it remains constant if reformulated as in Eq. (3.17) after cancellations have been accounted for. Similar remarks apply at higher order [cf. Eq. (3.18)].

As follows from these calculations, the cancellations are very significant, as they occur in the most singular part on the right-hand sides in Eqs. (2.29) and (2.31). Within a numerical implementation, this amounts to calculating relatively small numbers (e.g., the Fourier coefficients of $\left.T_{n}(g)[\xi]\right)$ as a difference of very large numbers [e.g., the Fourier coefficients of each (singular) individual term in the recurrence], which is, of course, a recipe for ill-conditioning (see Fig. 2).

\section{Effect of Cancellations in High-Order Implementations}

In connection with calculations of arbitrary order, the arguments in the preceding sections also suggest that this ill-conditioning should increase with increasing interface roughness as the terms in the recursions become more singular. Moreover, even for very smooth profiles, the instabilities should be enhanced as the number of Fourier modes in a calculation are increased, since the small contributions to high wave numbers are still attained as differences of large numbers (e.g., as in Fig. 2). The next series of numerical experiments (Figs. 3 and 4) were designed to confirm these predictions. To this end, we selected scattering profiles given by

$$
\begin{aligned}
& g_{s}(x)=\cos (x), \\
& g_{r}(x)=\left(2 \times 10^{-4}\right) x^{4}(2 \pi-x)^{4}-c_{0},
\end{aligned}
$$

$$
g_{L}(x)=\left\{\begin{array}{lc}
-\frac{2}{\pi} x+1, & 0 \leqslant x \leqslant \pi \\
\frac{2}{\pi} x-3, & \pi \leqslant x \leqslant 2 \pi
\end{array},\right.
$$

with decreasing regularity: $g_{s}$ is "smooth" (analytic), $g_{r}$ is "rough" (i.e., finite smoothness; four but not five times 
continuously differentiable), and $g_{L}$ is only Lipschitz continuous (it possesses a discontinuous first derivative). The constant $c_{0}$ in Eq. (3.21b) was chosen so that $g_{r}$ has zero mean (as do $g_{s}$ and $g_{L}$ ). To facilitate a comparison, we chose the scaling of each of the profiles so that they all have approximate amplitudes of 2 and maximum slopes of approximately 1 . The Fourier-series representations of $g_{r}$ and $g_{L}$ are given by

$$
\begin{aligned}
& g_{r}(x)=\sum_{k=1}^{\infty} \frac{96\left(2 k^{2} \pi^{2}-21\right)}{125 k^{8}} \cos (k x), \\
& g_{L}(x)=\sum_{k=1}^{\infty} \frac{8}{\pi^{2}(2 k-1)^{2}} \cos [(2 k-1) x],
\end{aligned}
$$

and, to minimize the effects of aliasing errors, we approximate them by their truncated Fourier series:

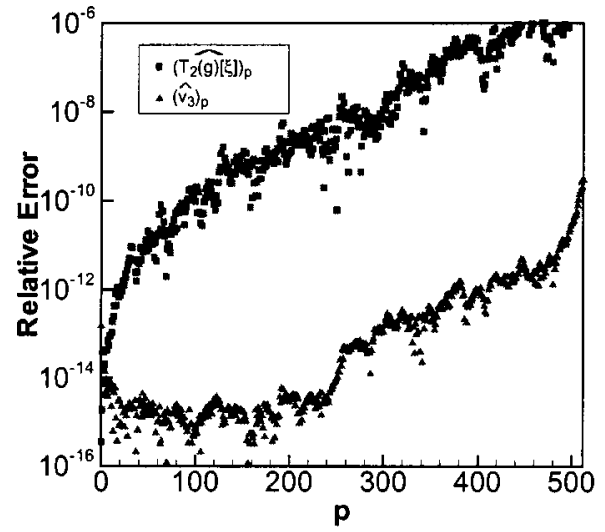

(a) $\alpha=\beta=1, k=\sqrt{2}$

$$
\begin{aligned}
& g_{r, P}(x)=\sum_{k=1}^{P} \frac{96\left(2 k^{2} \pi^{2}-21\right)}{125 k^{8}} \cos (k x), \\
& g_{L, P}(x)=\sum_{k=1}^{P / 2} \frac{8}{\pi^{2}(2 k-1)^{2}} \cos [(2 k-1)] .
\end{aligned}
$$

Indeed, if $P \ll N_{x} / 2$, the number of modes in our numerical approximation, the effects of aliasing will be minimal (in fact, no aliasing occurs if $n P+F \leqslant N_{x} / 2$, where $n$ is the degree of the perturbation series and $F$ is the number of Fourier modes in the Dirichlet data).

Figures 3 and 4 display the errors associated with perturbative approximations to surface currents (DNO) induced on the profiles in Eqs. (3.21). The figures show errors from approximations of increasing order corresponding to the $\mathrm{OE}$ and PFE formalisms as in Eq. (2.29) and Eqs. (2.8) and (2.31), respectively. More precisely, the errors correspond to approximations of the exact solutions (2.23), for which we can explicitly compute [cf. Eq. (2.24)]

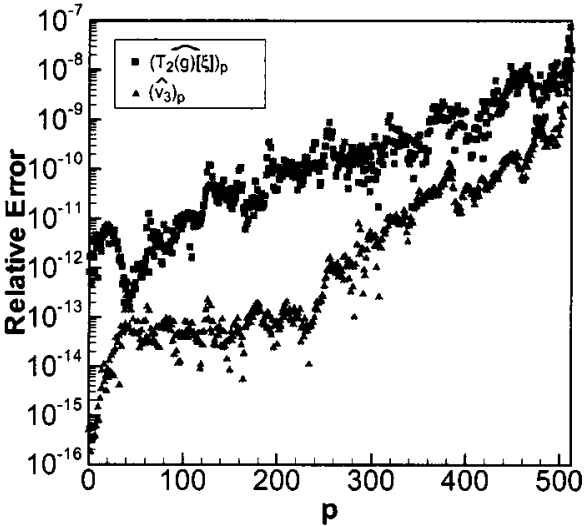

(b) $\alpha=\beta=100, k=100 \sqrt{2}$

Fig. 2. Plots of relative error in (absolute value of) the Fourier coefficients $\left(\widehat{T_{2}(g)[\xi]}\right)_{p}$ and $\left(\hat{v}_{3}\right)_{p}$ for different values of the wave number (and oblique incidence) as computed from the original OE and PFE recursions, respectively [cf. Eqs. (3.1c) and (3.16)]; the results are for rough profile $g$ and incidence $\xi$ with $\hat{g}_{p}=\hat{\xi}_{p}=p^{-2}$ for $|p| \leqslant F$. To avoid aliasing effects and isolate the instabilities, here $F$ was chosen as $F=N_{x} / 3$ for $T_{2}$ and $F=N_{x} / 4$ for $v_{3}$, where $N_{x}=2048$ is the number of discretization points.

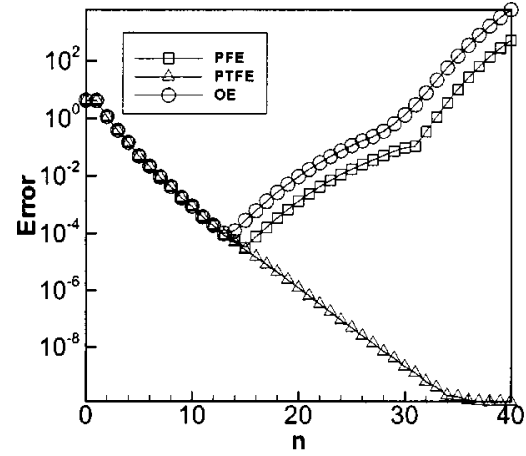

(a) Sinusoid

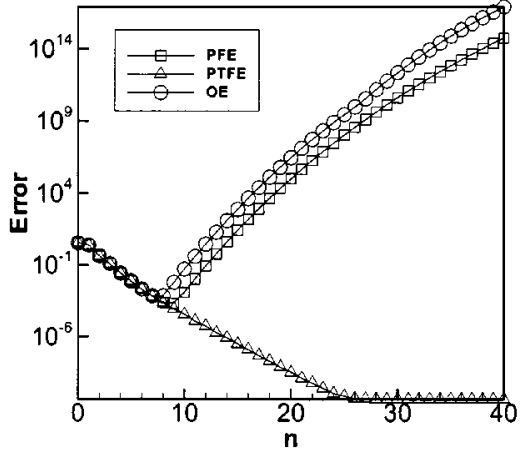

(b) Rough

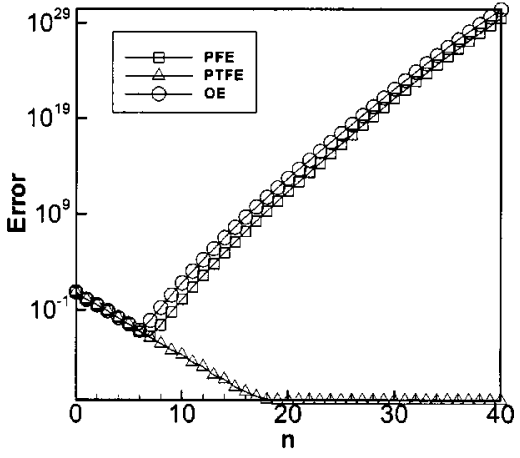

(c) Lipschitz

Fig. 3. Maximum error [cf. Eq. (3.23)] in the evaluation of the DNO with the OE, PFE, and PTFE algorithms. A comparison is given with exact solution (3.22) and $p=1$ for normal incidence with a wavelength-to-period ratio of 0.4368. (a) Sinusoidal profile (3.21a) ( $\left.\delta=0.09, N_{x}=64\right)$, (b) "rough" profile (3.21b) $\left(\delta=0.03, N_{x}=256\right)$, (c) Lipschitz profile $(3.21 \mathrm{c})\left(\delta=0.03, N_{x}=256\right)$. 


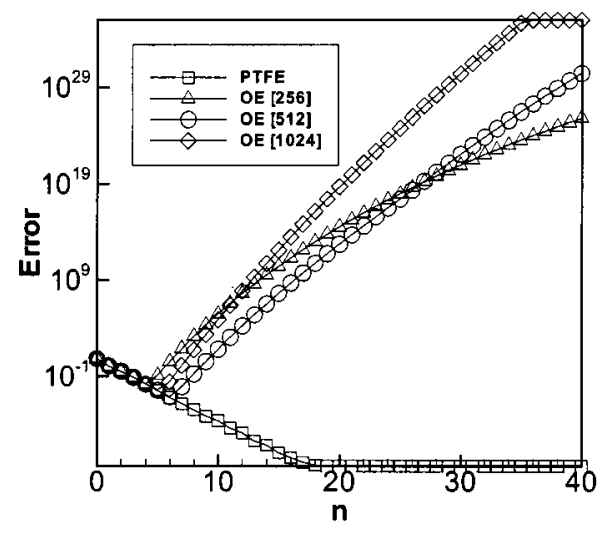

(a) OE: Several discretizations

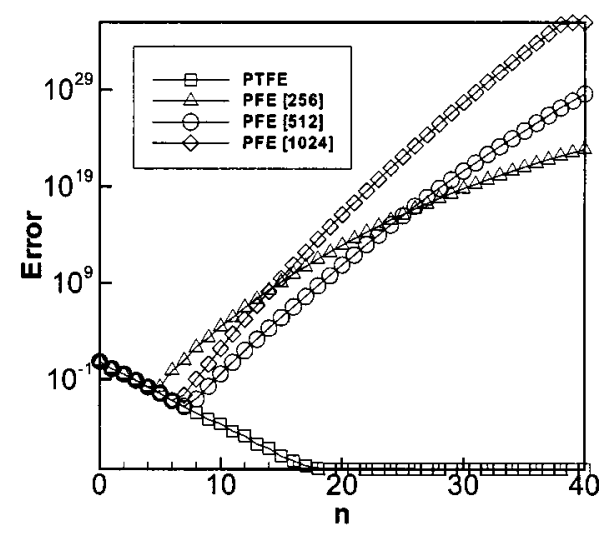

(b) PFE: Several discretizations

Fig. 4. Maximum error [cf. Eq. (3.23)] in the evaluation of the DNO with the OE, PFE, and PTFE algorithms. A comparison is given with exact solution (3.22) and $p=1$ for normal incidence with a wavelength-to-period ratio of 0.4368 . The effect of different discretizations for Lipschitz profile (3.21c) is given, showing the existence of an optimal number of discretization points $\left(N_{x}=512\right)$, a compromise between aliasing and conditioning errors: (a) effect on $\mathrm{OE}$ scheme, (b) effect on PFE algorithm.

$$
\begin{aligned}
T(g)\left[\exp \left\{i \alpha_{p} x+i \beta_{p} g(x)\right\}\right] & \\
& =\left[i \alpha_{p} \partial_{x} g(x)-i \beta_{p}\right] \exp \left[i \alpha_{p} x+i \beta_{p} g(x)\right] .
\end{aligned}
$$

As explained in Subsections 2.B and 2.C, the approximations are computed by using (fast) Fourier collocation on $N_{x}$ equally spaced nodes $x_{j}$ on $[0, d] .{ }^{57}$ When truncated and summed up to order $n$, this approach produces an approximation $T_{n, N_{x}}^{\text {approx }}$ for which the error is defined as

$$
\begin{aligned}
\text { Error } & =\operatorname{Error}\left(n, N_{x}\right) \\
& =\max _{1 \leqslant j \leqslant N_{x}}\left|T(g)[\xi]\left(x_{j}\right)-T_{n, N_{x}}^{\text {approx }}\left(x_{j}\right)\right| .
\end{aligned}
$$

The figures display this error as a function of $n$, the number of terms retained in the Taylor series, for each of the profiles $\left(P=40, \delta=0.09\right.$ for $g_{s}$ and $\delta=0.03$ for $g_{r}$ and $\left.g_{L}\right)$. For comparison purposes, the figures also display the results corresponding to a more stable algorithm, labeled partial transformed field expansions (PTFEs). This latter scheme corresponds to the implementation of alternative recursions that can be obtained much as in the $\mathrm{PFE}$ approach but with the addition of a nonlinear transformation effected a priori of the expansion (thus the PTFE name). As we shall see, our theoretical results in Section 4 guarantee that the transformation implicitly accounts for all cancellations and thus they predict the observed enhanced stability of the method.

The effect of the ill-conditioning of the OE and PFE formulas is evidenced in Fig. 3 and 4 in the form of an explosive divergence of the series beyond a few terms. Moreover, as predicted, the onset of this divergence is precipitated by a profile's roughness. Indeed, the figures show that as the profile is varied from smooth to rough to Lipschitz, the onset of divergence for the $\mathrm{OE}$ approach changes from $n=13$ to 7 to 6 while that for the PFE procedure occurs at $n=15,9$, and 7, respectively (note that, in contrast, the results of PTFE are consistently stable). A further refinement of the discretization (from $N_{x}=64$ for $g_{s}$ and $N_{x}=256$ for $g_{r}$ ) results in a loss of accuracy for both the smooth and rough profiles, indicating that conditioning errors overcome those that may arise from aliasing. For the Lipschitz profile, on the other hand, a discretization with $N_{x}=512$ actually produces slightly better results, but they again deteriorate at $N_{x}=1024$; see Fig. 4.

\section{CONVERGENCE}

As we demonstrated in Section 3, the cancellations appear at every order $n$ and, in fact, they become more severe with increasing $n$. In this section, we address the consequences of these observations on attempts at establishing the convergence of the perturbation series. Indeed, we shall first explain how what is perhaps the most natural approach cannot succeed, precisely on account of cancellations. In this connection, it can be argued that it is the failure of this approach that largely contributed to the historical confusion over the convergence of shapeperturbation expansions. We shall further show here that a strategy, based on a change of independent variables, can be devised to implicitly account for cancellations in a manner that then allows for a rather straightforward proof of the convergence of the perturbation series.

To begin, we note that a most natural attempt at the convergence of the series (2.7) or (2.22) would be to try to estimate the growth of the terms in the series inductively, appealing to the corresponding recursions. Indeed, if we could show that the size of the $n$th term in the series is bounded by $B^{n}$, for some constant $B$, then the existence of a positive radius of convergence will follow. Now, rather clearly, such a procedure cannot succeed, as any attempt at estimating the right-hand sides of the recursions will destroy the cancellations. This, in fact, can be easily illustrated within the Fourier version (2.13) of Eqs. (2.8) [a similar calculation can be done for Eq. (2.29)]. To this end, consider an arbitrary $M$-mode Fourier profile

$$
g(x)=\sum_{p=-M}^{M} \hat{g}_{p} \exp (i p x),
$$

and define $C_{l, p}$ by

$$
\frac{[g(x)]^{l}}{l !}=\sum_{p=-l M}^{l M} C_{l, p} \exp (i p x) .
$$


(a)

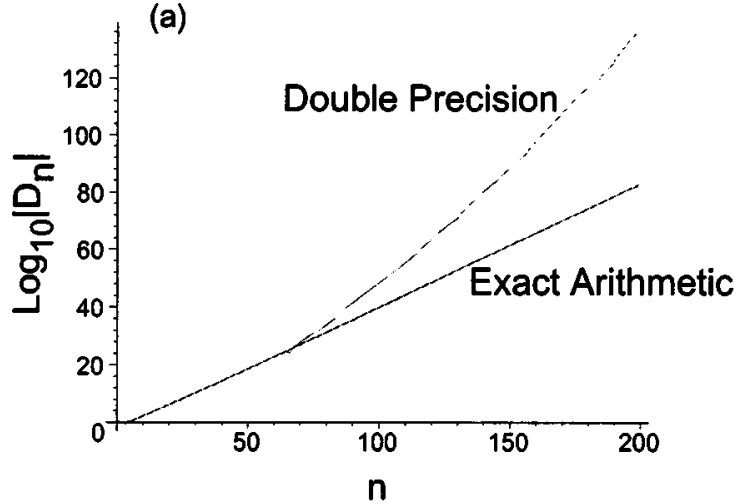

(b)

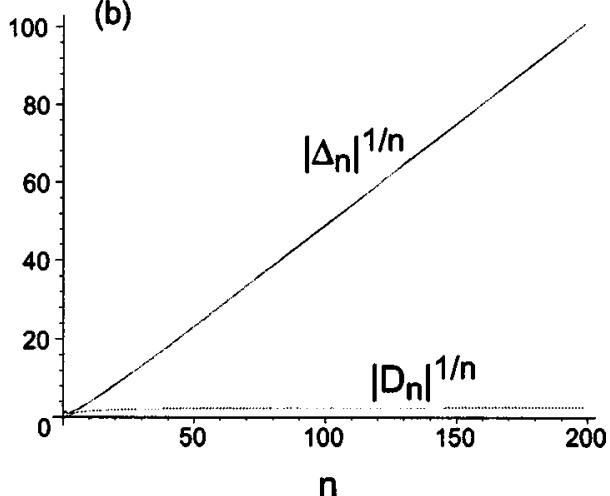

Fig. 5. (a) Results of the implementation of the recursion (4.1) in exact and finite-precision arithmetic, (b) root test for the sequences $D_{n}$ and $\Delta_{n}$ in Eqs. (4.1) and (4.2), respectively.

Then, from Eq. (2.13), it follows that

$$
D_{n}=-(-i \beta)^{n} C_{n, n M}-\sum_{l=0}^{n-1} C_{n-l,(n-l) M}\left(i \beta_{l M}\right)^{n-l} D_{l},
$$

where $D_{l}$ is the amplitude of the highest-order Fourier mode at order $l$ :

$$
D_{l}=d_{l, l M}
$$

In Fig. 5, we show some numerical results obtained from implementation of Eq. (4.1) in the most favorable case of an analytic boundary

$$
g(x)=2 \cos (x)=\exp (i x)+\exp (-i x) \quad(M=1) ;
$$

the wave number is $k=1$, and the incidence is normal. Figure 5(a) shows the effect of cancellations on the accuracy that can be attained: At sufficiently high orders, round-off errors completely overcome the values of interest. These errors, of course, arise from computing smaller values (i.e., the values of $D_{n}$ ) as differences of larger numbers [i.e., the individual terms on the righthand side of Eq. (4.1)]. The fact that these large numbers do indeed significantly cancel out is further demonstrated in Fig. 5(b), which also exemplifies the effect of straightforward bounds on the recursion. For this, we introduce the majorizing sequence $\Delta_{n}$, defined by the recurrence

$$
\Delta_{n}=|\beta|^{n}\left|C_{n, n M}\right|+\sum_{l=0}^{n-1}\left|C_{n-l,(n-l) M}\right|\left|\beta_{l M}\right|^{n-l} \Delta_{l} .
$$

Clearly,

$$
\left|D_{n}\right| \leqslant \Delta_{n},
$$

as Eq. (4.2) can be obtained from Eq. (4.1) upon use of the triangle inequality. As we show in Fig. 5(b), this estimate, which destroys the cancellations in Eq. (4.1), leads to a series with a vanishing radius of convergence. ${ }^{58}$

Interestingly, however, a direct estimation of the terms of the series can be achieved if a change of independent variables is effected before the perturbation expansion. Indeed, consider the transformation

$$
x^{\prime}=x, \quad y^{\prime}=a\left[\frac{y-g(x)}{a-g(x)}\right],
$$

which maps the domain $g(x)<y<a$ to the strip 0 $<y^{\prime}<a$. Problem (2.5) then transforms into

$$
\begin{aligned}
& \Delta^{\prime} u+k^{2} u=F\left(x^{\prime}, y^{\prime}\right), \\
& u\left(x^{\prime}, 0\right)=-\exp \left[i \alpha x^{\prime}-i \beta g\left(x^{\prime}\right)\right], \\
& \partial_{y^{\prime}} u\left(x^{\prime}, a\right)+T(a)[u(\cdot, a)]\left(x^{\prime}\right)=R\left(x^{\prime}\right), \\
& u\left(x^{\prime}+d, y^{\prime}\right)=\exp (i \alpha d) u\left(x^{\prime}, y^{\prime}\right)
\end{aligned}
$$

for $0<y^{\prime}<a$, where

$$
\begin{aligned}
& u\left(x^{\prime}, y^{\prime}\right)=v\left(x^{\prime},\{[a-g(x)] / a\} y^{\prime}+g(x)\right), \\
& F\left(x^{\prime}, y^{\prime}\right)=\operatorname{div}_{x^{\prime}}\left\{\frac{2 g\left(x^{\prime}\right)}{a} \nabla_{x^{\prime}} u-\frac{\left[g\left(x^{\prime}\right)\right]^{2}}{a^{2}} \nabla_{x^{\prime}} u\right. \\
& +\frac{\left(a-y^{\prime}\right) \nabla_{x^{\prime}} g\left(x^{\prime}\right)}{a} \partial_{y^{\prime}} u \\
& \left.-\frac{\left(a-y^{\prime}\right) g\left(x^{\prime}\right) \nabla_{x^{\prime}} g\left(x^{\prime}\right)}{a^{2}} \partial_{y^{\prime}} u\right\} \\
& +\partial_{y^{\prime}}\left[\frac{\left(a-y^{\prime}\right) \nabla_{x^{\prime}} g\left(x^{\prime}\right)}{a} \cdot \nabla_{x^{\prime}} u\right. \\
& -\frac{\left(a-y^{\prime}\right) g\left(x^{\prime}\right) \nabla_{x^{\prime}} g\left(x^{\prime}\right)}{a^{2}} \cdot \nabla_{x^{\prime}} u \\
& \left.-\frac{\left(a-y^{\prime}\right)^{2}\left|\nabla_{x^{\prime}} g\left(x^{\prime}\right)\right|^{2}}{a^{2}} \partial_{y^{\prime}} u\right] \\
& -\frac{\nabla_{x^{\prime}} g\left(x^{\prime}\right)}{a} \cdot \nabla_{x^{\prime}} u \\
& +\frac{g\left(x^{\prime}\right) \nabla_{x^{\prime}} g\left(x^{\prime}\right)}{a^{2}} \cdot \nabla_{x^{\prime}} u \\
& +\frac{\left(a-y^{\prime}\right)\left|\nabla_{x^{\prime}} g\left(x^{\prime}\right)\right|^{2}}{a^{2}} \partial_{y^{\prime}} u \\
& +\frac{2 g\left(x^{\prime}\right) k^{2}}{a} u-\frac{\left[g\left(x^{\prime}\right)\right]^{2} k^{2}}{a^{2}} u, \\
& R\left(x^{\prime}\right)=\frac{g\left(x^{\prime}\right)}{a} T(a)[u(\cdot, a)]\left(x^{\prime}\right) .
\end{aligned}
$$


Note that, coincidentally, this change of independent variables agrees with that used in the $\mathrm{C}$ method of Chandezon et al. ${ }^{59,60}$ Our use of the transformation, however, differs from that of the $\mathrm{C}$ method, where it is introduced to conveniently recast Eqs. (4.4) in the form of an eigenvalue problem for the vertical propagation constants. In contrast, our appeal to the transformation (4.3) is based on an entirely different property, specifically related to conditioning and convergence of shape-perturbation approaches. Indeed, dropping primes and formally expanding

$$
u(x, y)=\sum_{n=0}^{\infty} u_{n}(x, y),
$$

where $u_{n}=\mathcal{O}\left(g(x)^{n}\right)$, we obtain

$$
\begin{aligned}
& \Delta u_{n}+k^{2} u_{n}=\left(1-\delta_{n, 0}\right) F_{n}(x, y), \\
& u_{n}(x, 0)=-(-i \beta)^{n} \exp (i \alpha x) \frac{[g(x)]^{n}}{n !}, \\
& \partial_{y} u_{n}(x, a)+T(a)[u(\cdot, a)](x)=R_{n}(x), \\
& u_{n}(x+d, y)=\exp (i \alpha d) u_{n}(x, y)
\end{aligned}
$$

for $0<y<a$, where $\delta_{n, j}$ is the Kronecker delta,

$$
\begin{aligned}
& F_{n}(x, y)=\operatorname{div}_{x}\left[F_{n}^{(1)}(x, y)\right]+\partial_{y} F_{n}^{(2)}(x, y)+F_{n}^{(3)}(x, y) \text {, } \\
& F_{n}^{(1)}(x, y)=\frac{2 g(x)}{a} \nabla_{x} u_{n-1}-\frac{[g(x)]^{2}}{a^{2}} \nabla_{x} u_{n-2} \\
& +\frac{(a-y) \nabla_{x} g(x)}{a} \partial_{y} u_{n-1} \\
& -\frac{(a-y) g(x) \nabla_{x} g(x)}{a^{2}} \partial_{y} u_{n-2}, \\
& F_{n}^{(2)}(x, y)=\frac{(a-y) \nabla_{x} g(x)}{a} \cdot \nabla_{x} u_{n-1} \\
& -\frac{(a-y) g(x) \nabla_{x} g(x)}{a^{2}} \cdot \nabla_{x} u_{n-2} \\
& -\frac{(a-y)^{2}\left|\nabla_{x} g(x)\right|^{2}}{a^{2}} \partial_{y} u_{n-2}, \\
& F_{n}^{(3)}(x, y)=-\frac{\nabla_{x} g(x)}{a} \cdot \nabla_{x} u_{n-1}+\frac{g(x) \nabla_{x} g(x)}{a^{2}} \cdot \nabla_{x} u_{n-2} \\
& +\frac{(a-y)\left|\nabla_{x} g(x)\right|^{2}}{a^{2}} \partial_{y} u_{n-2}+\frac{2 g(x) k^{2}}{a} u_{n-1} \\
& -\frac{[g(x)]^{2} k^{2}}{a^{2}} u_{n-2}, \\
& R_{n}(x)=\frac{g(x)}{a} T(a)\left[u_{n-1}(\cdot, a)\right](x) .
\end{aligned}
$$

Equations (4.6) are obviously similar in structure to Eqs. (2.8). There are, however, significant differences. Most notably, in contrast with Eqs. (2.8), Eqs. (4.6) do not involve high-order derivatives of lower-order iterates $\left\{u_{l}\right\}_{l=0}^{n-1}$, and thus they do not require derivatives of the profile $g(x)$ of increasingly high order. In fact, at this point, under the sole assumption that $g(x)$ has two continuous derivatives, it can be inductively shown, by using the techniques of Ref. 55, that

$$
\left\|u_{n}\right\|_{2} \leqslant K_{1} B^{n}
$$

for some constants $K_{1}, B>0$, where the (Sobolev) norm of index $s$ is defined by

$$
\|u\|_{s}^{2}=\sum_{l=0}^{s} \sum_{p=-\infty}^{\infty}\left(1+|p|^{2}\right)^{s-l} \int_{0}^{a}\left|\partial_{y}^{l} \hat{u}_{p}(y)\right|^{2} \mathrm{~d} y .
$$

Indeed, in contrast with the solutions to Eqs. (2.8), the solutions $u_{n}$ to Eqs. (4.6) can be iteratively bounded in these Sobolev norms with a direct use of the triangle inequality on the right-hand sides $F_{n}$ and $R_{n}$ that depend on $\left\{u_{l}\right\}_{l=0}^{n-1}$. Such a strategy readily delivers inequality (4.7), which, in particular, demonstrates that the transformed field $u$ is analytic with respect to the perturbation parameter $\delta$ under rather permissive smoothness restrictions on $g(x)$.

In more detail, classical results from the theory of elliptic partial differential equations ${ }^{61,62}$ indicate that if $\phi(x, y), \mu(x)$, and $\eta(x)$ are such that

$$
\begin{aligned}
\|\phi\|_{0} & =\sum_{p=-\infty}^{\infty} \int_{0}^{a}\left|\hat{\phi}_{p}(y)\right|^{2} \mathrm{~d} y, \\
\|\mu\|_{1 / 2} & =\sum_{p=-\infty}^{\infty}\left(1+|p|^{2}\right)^{1 / 2}\left|\hat{\mu}_{p}\right|^{2}, \\
\|\eta\|_{3 / 2} & =\sum_{p=-\infty}^{\infty}\left(1+|p|^{2}\right)^{3 / 2}\left|\hat{\eta}_{p}\right|^{2}
\end{aligned}
$$

are all bounded, then there exists a unique solution of

$$
\begin{aligned}
& \Delta w(x, y)+k^{2} w(x, y)=\phi(x, y), \\
& w(x, 0)=\eta(x), \\
& \partial_{y} w(x, a)+T(a)[w(\cdot, a)](x)=\mu(x), \\
& w(x+d, y)=\exp (i \alpha d) w(x, y)
\end{aligned}
$$

for $0<y<a$ satisfying

$$
\|w\|_{2} \leqslant K_{e}\left(\|\phi\|_{0}+\|\eta\|_{3 / 2}+\|\mu\|_{1 / 2}\right)
$$

for some $K_{e}>0$. Now, the explicit solution of Eqs. (4.6) at order 0 verifies the inequality (4.7) for $n=0$. On the other hand, if we assume the inequality (4.7) for all $n$ $<N$, it can be shown, again as in Refs. 52 and 55, by using the triangle inequality, that

$$
\begin{aligned}
\left\|F_{N}\right\|_{0} & \leqslant K_{1} K_{0}\left(C_{g} B^{N-1}+C_{g}^{2} B^{N-2}\right), \\
\left\|R_{N}\right\|_{1 / 2} & \leqslant K_{1} K_{0} C_{g} B^{N-1},
\end{aligned}
$$

where $K_{0}>0$, and $C_{g}>0$ is a constant bounding the second (continuous) derivative of $g(x)$ on $[0, d]$. Then, from inequalities (4.9) and (4.10), we have 


$$
\begin{aligned}
\left\|u_{N}\right\|_{2} \leqslant & K_{e}\left\{\left\|F_{N}\right\|_{0}+\left\|-(i \beta)^{N} \exp (i \alpha x) \frac{[g(x)]^{N}}{N !}\right\|_{3 / 2}\right. \\
& \left.+\left\|R_{N}\right\|_{1 / 2}\right\} \\
\leqslant & K_{e}\left[K_{1} K_{0}\left(C_{g} B^{N-1}+C_{g}^{2} B^{N-2}\right)+\beta^{N} \frac{C_{g}^{N}}{N !}\right. \\
& \left.+K_{1} K_{0} C_{g} B^{N-1}\right] \leqslant K_{1} B^{N}
\end{aligned}
$$

if we choose $B>C_{g} \max \left\{6 K_{e} K_{0}, \sqrt{3 K_{e} K_{0}}\right.$, $\left.\left[3 K_{e} /\left(K_{1} N !\right)\right]^{1 / N} \beta\right\}$, which, by induction, proves the inequality (4.7). We remark again that the success of this argument relied on the possibility of bounding the norms of $F_{n}$ and $R_{n}$ by the sum of the norms of each of their constitutive terms to obtain the inequalities (4.10), a possibility that, in turn, relies on the absence of cancellations in these sums.

Finally, by similar arguments, ${ }^{52}$ it can be shown that if $g(x)$ is analytic, then

$$
\left\|\frac{\partial_{x}^{l} \partial_{y}^{m}}{l ! m !} u_{n}\right\|_{2} \leqslant C_{2} B^{n} \frac{D^{m}}{(m+1)^{2}} \frac{A^{l}}{(l+1)^{2}},
$$

which, in particular, implies that $u$ is jointly analytic with respect to $\delta$ and the spatial variables $x$ and $y$. If we recall the change of variables (4.3) that produced $u$ from $v$ in Eq. (4.5), it then follows that the original field $v$ itself enjoys this joint analyticity property. This latter observation, in turn, guarantees the validity of the recurrences, and it also recovers the original result of Ref. 23 in a direct manner, bypassing any reference to the integral equation formulation.

\section{CONCLUDING REMARKS}

In this paper, we have analyzed the conditioning properties of shape-deformation methods for rough-surfacescattering simulations. In particular, we have presented a theory that explains the observed behavior of these schemes, whose performance typically deteriorates with increasing surface roughness and/or increasing number of discretization points beyond a threshold. The theory attributes this behavior to significant cancellations in the recursions underlying these numerical procedures. A variety of analytical and numerical results, encompassing both low- and high-order calculations, were presented that support this contention.

Our discussion also addressed theoretical and numerical consequences of these cancellations. On the theoretical side, we showed how they prevent a most straightforward proof of convergence of the perturbation series, and we suggested that this significantly added to the historical controversy over the properties of the series. Moreover, we also showed that a simple change of independent variables can be used to rectify the cancellations and allow for a new, direct demonstration of convergence. As for numerical implementations, we showed that the cancellations are always significant and that they become more pronounced with decreasing surface regularity. In particular, even for smooth profiles, the cancellations impose certain restrictions on the discretizations (in either physical or Fourier space), as these must balance accuracy requirements, which demand a large number of points, with stability, which favors a small number of frequencies.

Finally, our results can be interpreted as providing guidelines for the design of new, stabilized implementations of boundary perturbation methods. Indeed, our very precise identification of the origins of instabilities can be used in attempting to mollify them. In the case of low- (first-, second-) order calculations, the relative simplicity of the recurrence could potentially allow for an $e x$ plicit account of cancellations, that is, for a restatement of the formulas in a manner that explicitly avoids the calculation of small numbers (e.g., high-frequency Fourier coefficients) as differences of large ones. For higher-order simulations, on the other hand, this strategy becomes increasingly complex, as the formulas get progressively more complicated. In this case, however, our theoretical results suggest a number of schemes to implicitly account for cancellations. Indeed, as we stated, the success of our approach to convergence of the perturbation series implies that the newly derived recursions in the transformed variables do not exhibit significant cancellations and thus they must lead to a well-conditioned scheme (in fact, the curves labeled PTFE in Figs. 3 and 4, which precisely correspond to such a scheme, provide some evidence to support this assertion). More generally, a more fundamental property of the transformed recursions that could prove useful in the design of new implementations is that, in contrast with the original recurrences, they are derived without the need to differentiate the fields across the boundary of their domains of definition. Indeed, it is this characteristic that eventually leads to formulas that do not involve high-order derivatives of the surface [cf. Eqs. (4.6)] and that, more generally, could guide the design of alternative stabilized schemes.

\section{APPENDIX A: SMOOTHNESS OF REMAINDERS IN LOW-ORDER OPERATOR EXPANSION AND PARTIAL FIELD EXPANSION FORMULAS}

For the sake of completeness, in this appendix we present a discussion on the regularity properties of the operators $R_{f}$ and $S_{f}$ introduced in Subsection 3.A [cf. Eqs. (3.2) and (3.6), respectively]. Specifically, we have the following:

Theorem A.1. Let $f(x)$ be a $d$-periodic and Lipschitzcontinuous function, and let $\eta(x)$ be quasi-periodic and square-integrable on $[0, d]$. Define $R_{f}[\eta]$ by

$$
R_{f}[\eta]=\beta_{D}[f \eta]-f \beta_{D}[\eta] .
$$

Then $R_{f}[\eta]$ is square-integrable on $[0, d]$.

Note that this result implies that $R_{f}[\eta]$ is more regular than either $\beta_{D}[f \eta]$ or $f \beta_{D}[\eta]$.

Proof. We begin by introducing the function 


$$
Q(x)=\sum_{p=-\infty}^{\infty} \beta_{p} \exp \left(i \alpha_{p} x\right)
$$

where the sum is to be understood in the sense of Abel. ${ }^{63}$ We note that the function $Q$ has a (periodic) quadratic singularity at $x=0$. In fact, from the asymptotic expansion

$$
\begin{gathered}
\beta_{p}=i|p|+i \alpha \operatorname{sgn}(p)-i \frac{k^{2}}{2|p|}+O\left(p^{-2}\right) \\
\quad \text { as } p \rightarrow \infty
\end{gathered}
$$

it is easy to see that

$$
\begin{aligned}
Q(x)= & \exp (i \alpha x)\left\{-\frac{i}{2} \frac{1}{\sin ^{2}(\pi x / d)}-\alpha \frac{\cos (\pi x / d)}{\sin (\pi x / d)}\right. \\
+ & \left.\frac{i k^{2}}{2} \ln \left[\sin ^{2}(\pi x / d)\right]+P(x)\right\},
\end{aligned}
$$

where $P(x)$ is bounded. Then, expanding

$$
\eta(x)=\sum_{p=-\infty}^{\infty} \hat{\eta}_{p} \exp \left(i \alpha_{p} x\right)
$$

and using the definition (2.27), we have that

$$
\begin{aligned}
\beta_{D}[\eta](x) & =\sum_{p=-\infty}^{\infty} \beta_{p} \hat{\eta}_{p} \exp \left(i \alpha_{p} x\right) \\
& =\sum_{p=-\infty}^{\infty} \beta_{p} \exp \left(i \alpha_{p} x\right) \frac{1}{d} \int_{0}^{d} \eta(y) \exp \left(-i \alpha_{p} y\right) \mathrm{d} y \\
& =\frac{1}{d} \int_{0}^{d} \eta(y) Q(x-y) \mathrm{d} y
\end{aligned}
$$

and then, from Eq. (A1), it follows that

$$
\begin{aligned}
R_{f}[\eta](x) & =\beta_{D}[f \eta]-f \beta_{D}[\eta] \\
& =\frac{1}{d} \int_{0}^{d} \eta(y)[f(y)-f(x)] Q(x-y) \mathrm{d} y
\end{aligned}
$$

Finally, since $f$ is Lipschitz continuous, the last integral is precisely a (nonconvolution) singular integral in the sense that the singularity in the kernel

$$
[f(y)-f(x)] Q(x-y)
$$

behaves like $1 / \sin [\pi(x-y) / d]$ [cf. Eq. (A2)]. The square integrability of $R(f)[\eta]$ then follows from the classical theory of singular-integral operators. ${ }^{64}$

Theorem A.2. Let $f(x)$ and $\eta$ be as in Theorem A.1, and define

$$
S_{f}[\eta]=-R_{f^{2} / 2}[\eta]+f R_{f}[\eta] .
$$

Then the function $\beta_{D} S_{f}[\eta]$ is square-integrable on $[0, d]$. Proof. From Eq. (A3), we have

$$
\begin{aligned}
S_{f}[\eta](x)= & \frac{1}{d} \int_{0}^{d} \eta(y) Q(x-y)(f(x)[f(y)-f(x)] \\
& \left.-\left\{\frac{[f(y)]^{2}}{2}-\frac{[f(x)]^{2}}{2}\right\}\right) \mathrm{d} y,
\end{aligned}
$$

which can be simply written as

$$
S_{f}[\eta](x)=-\frac{1}{2 d} \int_{0}^{d} \eta(y) Q(x-y)[f(y)-f(x)]^{2} \mathrm{~d} y .
$$

Then, again, classical results in singular-integral theory ${ }^{64}$ imply that a derivative of $S_{f}[\eta]$ [and thus the function $\left.\beta_{D} S_{f}[\eta]\right]$ will be square-integrable on account of the additional regularity of the kernel $Q(x-y)[f(y)-f(x)]^{2}$ in Eq. (A4).

\section{ACKNOWLEDGMENTS}

David P. Nicholls gratefully acknowledges support from the National Science Foundation (NSF) through grants DMS-0196452 and DMS-0139822. Fernando Reitich gratefully acknowledges support from the NSF through grants DMS-9971379 and DMS-0311763, from the U.S. Air Force Office of Scientific Research through contract F49620-02-1-0052, and from the U.S. Army High Performance Computing Research Center (AHPCRC) under U.S. Army Research Laboratory cooperative agreement DAAD19-01-2-0014.

Disclaimer. Effort sponsored by the Air Force Office of Scientific Research, Air Force Materials Command, USAF, under grant F49620-02-1-0052, and by the AHPCRC under the auspices of the Department of the Army, Army Research Laboratory cooperative agreement DAAD19-01-2-0014. The U.S. Government is authorized to reproduce and distribute reprints for governmental purposes notwithstanding any copyright notation thereon. The views and the conclusions contained herein are those of the author and should not be interpreted as necessarily representing the official policies or endorsements, either expressed or implied, of the Air Force Office of Scientific Research, the Army Research Laboratory, or the U.S. Government.

The authors' e-mail addresses are Nicholls.2@nd.edu and reitich@math.umn.edu.

\section{REFERENCES}

1. Lord Rayleigh, "On the dynamical theory of gratings," Proc. R. Soc. London Ser. A 79, 399-416 (1907).

2. S. O. Rice, "Reflection of electromagnetic waves from slightly rough surfaces," Commun. Pure Appl. Math. 4, 351-378 (1951).

3. G. V. Anand and M. K. George, "Normal mode sound propagation in an ocean with sinusoidal surface waves," J. Acoust. Soc. Am. 80, 238-243 (1986).

4. G. V. Anand and M. K. George, "Normal mode sound propagation in an ocean with random narrow-band surface waves," J. Acoust. Soc. Am. 94, 279-292 (1993).

5. E. Y. Harper and F. M. Labianca, "Perturbation theory for 
scattering of sound from a point source by a moving rough surface in the presence of refraction," J. Acoust. Soc. Am. 57, 1044-1051 (1975)

6. E. Y. Harper and F. M. Labianca, "Scattering of sound from a point source by a rough surface progressing over an isovelocity ocean," J. Acoust. Soc. Am. 58, 349-364 (1975).

7. W. A. Kuperman and F. F. Ingenito, "Attenuation of the coherent component of sound propagating in shallow water with rough boundaries," J. Acoust. Soc. Am. 61, 1178-1187 (1977).

8. A. H. Nayfeh and O. R. Asfar, "Parallel-plate waveguide with sinusoidally perturbed boundaries," J. Appl. Phys. 45, 4797-4800 (1974)

9. J. R. Wait, "Perturbation analysis for reflection from twodimensional periodic sea waves," Radio Sci. 6, 387-391 (1971).

10. W. C. Meecham, "On the use of the Kirchoff approximation for the solution of reflection problems," J. Rat. Mech. Anal. 5, 323-334 (1956)

11. J. L. Uretsky, "The scattering of plane waves from periodic surfaces," Ann. Phys. 33, 400-427 (1965).

12. J. M. Chesneaux and A. A. Wirgin, "Response to comments on 'Reflection from a corrugated surface revisited'," J. Acoust. Soc. Am. 98, 1815-1816 (1995).

13. J. J. Greffet, "Scattering of electromagnetic waves by rough dielectric surfaces," Phys. Rev. B 37, 6436-6441 (1988).

14. J. J. Greffet, C. Baylard, and P. Versaevel, "Diffraction of electromagnetic waves by crossed gratings: a series solution," Opt. Lett. 17, 1740-1742 (1992).

15. J. J. Greffet and Z. Maassarani, "Scattering of electromagnetic waves by a grating: a numerical evaluation of the iterative-series solution,” J. Opt. Soc. Am. A 7, 1483-1493 (1990).

16. D. R. Jackson, D. P. Winebrenner, and A. Ishimaru, "Comparison of perturbation theories for rough-surface scattering,” J. Acoust. Soc. Am. 83, 961-969 (1988).

17. L. Kazandjian, "Comparison of the Rayleigh-Fourier and extinction theorem methods applied to scattering and transmission at a rough solid-solid interface," J. Acoust. Soc. Am. 92, 1679-1691 (1992).

18. C. Lopez, F. J. Yndurain, and N. Garcia, "Iterative series for calculating the scattering of waves from hard corrugated surfaces,” Phys. Rev. B 18, 970-972 (1978).

19. A. A. Maradudin, "Iterative solutions for electromagnetic scattering by gratings," J. Opt. Soc. Am. 73, 759-764 (1983).

20. J. Roginsky, "Derivation of closed-form expressions for the $T$ matrices of Rayleigh-Rice and extinction-theorem perturbation theories," J. Acoust. Soc. Am. 90, 1130-1137 (1991).

21. V. I. Tatarskii, "Relation between the Rayleigh equation in diffraction theory and the equation based on Green's formula," J. Opt. Soc. Am. A 12, 1254-1260 (1995).

22. A. Wirgin, "Scattering from hard and soft corrugated surfaces: iterative corrections to the Kirchhoff approximation through the extinction theorem," J. Acoust. Soc. Am. 85, 670-679 (1989).

23. O. P. Bruno and F. Reitich, "Solution of a boundary value problem for the Helmholtz equation via variation of the boundary into the complex domain," Proc. R. Soc. Edinburgh Sect. A 122, 317-340 (1992)

24. O. P. Bruno and F. Reitich, "Numerical solution of diffraction problems: a method of variation of boundaries," J. Opt. Soc. Am. A 10, 1168-1175 (1993).

25. O. P. Bruno and F. Reitich, "Numerical solution of diffraction problems: a method of variation of boundaries. II. Finitely conducting gratings, Padé approximants, and singularities," J. Opt. Soc. Am. A 10, 2307-2316 (1993)

26. O. P. Bruno and F. Reitich, "Numerical solution of diffraction problems: a method of variation of boundaries. III. Doubly periodic gratings,” J. Opt. Soc. Am. A 10, 2551-2562 (1993).

27. O. P. Bruno and F. Reitich, "Calculation of electromagnetic scattering via boundary variations and analytic continuation," Appl. Comput. Electromagn. Soc. J. 11, 17-31 (1996).
28. O. P. Bruno and F. Reitich, "Boundary-variation solutions for bounded-obstacle scattering problems in three dimensions," J. Acoust. Soc. Am. 104, 2579-2583 (1998).

29. O. P. Bruno and F. Reitich, "High-order boundary perturbation methods," in Mathematical Modeling in Optical Science, Vol. 22 of Frontiers in Applied Mathematics (Society for Industrial and Applied Mathematics, Philadelphia, Pa., 2001), pp. 71-110.

30. D. M. Milder, "An improved formalism for rough-surface scattering of acoustic and electromagnetic waves," in Wave Propagation and Scattering in Varied Media II, V. Varadan, ed., Proc. SPIE 1558, 213-221 (1991).

31. D. M. Milder, "An improved formalism for wave scattering from rough surfaces,” J. Acoust. Soc. Am. 89, 529-541 (1991)

32. D. M. Milder and H. T. Sharp, "Efficient computation of rough surface scattering," in Mathematical and Numerical Aspects of Wave Propagation Phenomena (Society for Industrial and Applied Mathematics, Philadelphia, Pa., 1991), pp. 314-322.

33. D. M. Milder and H. T. Sharp, "An improved formalism for rough surface scattering. II: Numerical trials in three dimensions," J. Acoust. Soc. Am. 91, 2620-2626 (1992).

34. P. J. Kaczkowski and E. I. Thorsos, "Application of the operator expansion method to scattering from onedimensional moderately rough Dirichlet random surfaces," J. Acoust. Soc. Am. 96, 957-972 (1994).

35. D. M. Milder, "Role of the admittance operator in roughsurface scattering," J. Acoust. Soc. Am. 100, 759-768 (1996).

36. D. M. Milder, "An improved formalism for electromagnetic scattering from a perfectly conducting rough surface," Radio Sci. 31, 1369-1376 (1996).

37. R. A. Smith, "The operator expansion formalism for electromagnetic scattering from rough dielectric surfaces," Radio Sci. 31, 1377-1385 (1996)

38. A. G. Voronovich, Wave Scattering from Rough Surfaces, 2nd ed. (Springer-Verlag, Berlin, 1999).

39. K. F. Warnick and W. C. Chew, "Numerical simulation methods for rough surface scattering," Waves Random Media 11, R1-R30 (2001).

40. H. Hasimoto and H. Ono, "Nonlinear modulation of gravity waves," J. Phys. Soc. Jpn. 33, 805-811 (1972).

41. K. M. Watson and B. J. West, "A transport-equation description of nonlinear ocean surface wave interactions," J. Fluid Mech. 70, 815-826 (1975).

42. D. M. Milder, "The effects of truncation on surface-wave Hamiltonians," J. Fluid Mech. 217, 249-262 (1990).

43. W. Craig and C. Sulem, "Numerical simulation of gravity waves," J. Comput. Phys. 108, 73-83 (1993).

44. R. A. Smith, "An operator expansion formalism for nonlinear surface waves over variable depth,” J. Fluid Mech. 363, 333-347 (1998).

45. D. P. Nicholls, "Traveling gravity water waves in two and three dimensions," Ph.D. thesis (Brown University, Providence, R.I., 1998).

46. D. P. Nicholls, "Traveling water waves: spectral continuation methods with parallel implementation," J. Comput. Phys. 143, 224-240 (1998).

47. P. Dinesen and J. S. Hesthaven, "Fast and accurate modeling of waveguide grating couplers," J. Opt. Soc. Am. A 17 1565-1572 (2000).

48. P. Dinesen and J. S. Hesthaven, "Fast and accurate modeling of waveguide grating couplers. II. The threedimensional vectorial case," J. Opt. Soc. Am. A 18, 28762885 (2001).

49. G. Bao and D. Dobson, "Variational methods for diffractive optics modeling," in Mathematical Modeling in Optical Science, Vol. 22 of Frontiers in Applied Mathematics (Society for Industrial and Applied Mathematics, Philadelphia, Pa., 2001), pp. 37-70.

50. O. P. Bruno and L. A. Kunyansky, "A fast, high-order algorithm for the solution of surface scattering problems. I: Basic implementation, tests and applications," J. Comput. Phys. 169, 80-110 (2001). 
51. D. Colton and R. Kress, Inverse Acoustic and Electromagnetic Scattering Theory, 2nd ed. (Springer-Verlag, Berlin, 1998).

52. D. P. Nicholls and F. Reitich, "Analytic continuation of Dirichlet-Neumann operators," Numer. Math. 94, 107-146 (2003).

53. A. P. Calderón, "Cauchy integrals on Lipschitz curves and related operators," Proc. Natl. Acad. Sci. USA 75, 13241327 (1977).

54. R. Coifman and Y. Meyer, "Nonlinear harmonic analysis and analytic dependence," in Pseudodifferential Operators and Applications (American Mathematical Society, Providence, R.I., 1985), pp. 71-78.

55. D. P. Nicholls and F. Reitich, "A new approach to analyticity of Dirichlet-Neumann operators," Proc. R. Soc. Edinburgh, Sect. A 131, 1411-1433 (2001)

56. D. P. Nicholls and F. Reitich, "Stability of high-order perturbative methods for the computation of DirichletNeumann operators," J. Comput. Phys. 170, 276-298 (2001).

57. C. Canuto, M. Y. Hussaini, A. Quarteroni, and T. A. Zang,
Spectral Methods in Fluid Dynamics (Springer-Verlag, New York, 1988).

58. A. Friedman and F. Reitich, "Symmetry-breaking bifurcation of analytic solutions to free boundary problems: an application to a model of tumor growth," Trans. Am. Math. Soc. 353, 1587-1634 (2001).

59. J. Chandezon, D. Maystre, and G. Raoult, "A new theoretical method for diffraction gratings and its numerical application," J. Mod. Opt. 11, 235-241 (1980).

60. L. Li, J. Chandezon, G. Granet, and J. P. Plumey, "Rigorous and efficient grating-analysis method made easy for optical engineers," Appl. Opt. 38, 304-313 (1999).

61. O. A. Ladyzhenskaya and N. N. Ural'tseva, Linear and Quasilinear Elliptic Equations (Academic, New York, 1968).

62. L. C. Evans, Partial Differential Equations (American Mathematical Society, Providence, R.I., 1998).

63. P. L. Butzer and R. J. Nessel, Fourier Analysis and Approximation (Academic, New York, 1971).

64. E. M. Stein, Singular Integrals and Differentiability Properties of Functions (Princeton U. Press, Princeton, N.J., 1970). 